\title{
Study on Seismic Behaviors of the Pagoda in Songyue Temple
}

\author{
Shaochun $\mathrm{Ma}^{1,2, *}$, Lianghui $\mathrm{Li}^{1}$, Peng $\mathrm{Bao}^{1,2,{ }^{*}}$ \\ ${ }^{1}$ School of Civil Engineering and Architecture, Henan University, Kaifeng, Henan, 475004, China \\ ${ }^{2}$ Kaifeng Research Center for Engineering Repair and Material Recycle, Henan University, Henan, Kaifeng 475004, China
}

\begin{abstract}
The dynamic properties and anti-earthquake behaviors of Pagoda in Songyue Temple was studied by numerical simulation method. Firstly, based on the its contexture, the equivalent model reflecting the force transfer mechanism is established. Secondly, by analyzing the dynamic characteristics of the computational model, the vibration modes、 natural vibration frequency and natural period of vibration are measured. The correctness of the model verified by comparing the theoretical calculation results and the simulation results. Lastly, the seismic behaviors of the Pagoda in Songyue Temple was evaluated according to the elastic spectrum analysis and time-history analysis results and related data. The results show that: the stiffness is uniformly distributed and the seismic performance is good enough. Under the action of earthquake force, it is easy to produce tension rupture and the tacha the pagoda body without section change, as weak link of the structure, need to focus on.
\end{abstract}

\section{Introduction}

Pagoda in Songyue Temple is located in the south of Song Mountain which in Dengfeng. It was built in Northern Wei Dynasty (520-524 CE) [1]. It is one of the four famous ancient pagodas in China and is the only existing ancient pagoda with dodecahedron. The damage was caused by factors like earthquakes and vandalism, over the past millennium has reduced pagoda's bearing and deformation capacities. the unpredictable natural disaster will importantly influence the safety of it. A detailed analysis of its earthquake resistant mechanism is required to develop effective repair and restoration measures[2 3].

The ancient pagoda is a precious historical relic. It should avoide damaging external force when repaired and protected the pagoda. Therefore, evaluating the pagoda's seismic performance by numerical simulation method is adopted. The finite element model is built by Ansys according to contexture that simplified the modal. Through modal, response spectrum and time history analysis, dynamic response of Pagoda in Songyue Temple are studied. Based on the results of simulation calculation, evaluated the safety reliability and damage degree of the Pagoda under earthquake action, known that the seismic performance of Pagoda in Songyue Temple is good enough. According to the damage and weak links, it provides important basis datas for protection of Pagoda in Songyue Temple[4 5].

\section{The Modal of the Pagoda in Songyue Temple}

Pagoda in Songyue Temple, a total height of more than 37 meters. It is composed of abutment, pagoda body, fifteen floors with thick eaves and baocha. On the low wall between the eaves, kinds of doors and windows are built. the floors gradually decrease in diameter from bottom to top. Except for the bottom floor of the pagoda, the rest are changed to octagonal shape. The whole tower chamber is connected from top to bottom, which is cylindrical and regular in shape ( Figure 1). 


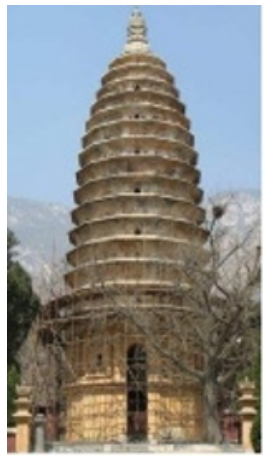

(a) Pagoda in Songyue Temple

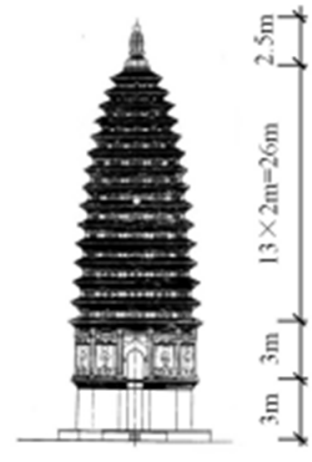

(b) Elevation

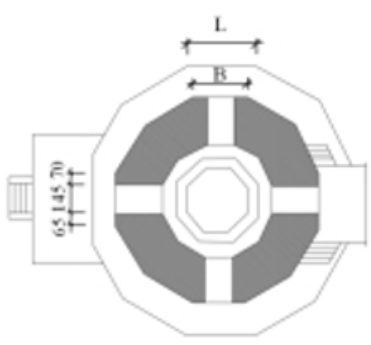

(c) Plan

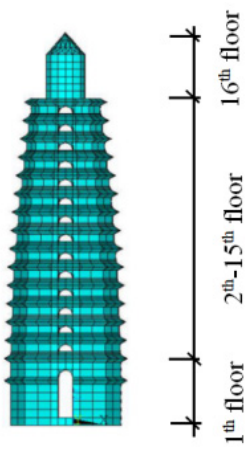

(d) The finite element modal

Figure 1. The structure of Pagoda in Songyue Temple

\section{Build the modal}

The pagoda body is divided into upper and lower parts by the waist eaves, and the upper part decrease in diameter from bottom to top. The ancient pagoda was decorated with columns at the corner of the lower parts. Since it did not affect the internal force transmission mechanism of the whole structure, its weight was dispersed to the first floor of brick eaves, which was set at a position of $4.59 \mathrm{~m}$ above the bottom, with $1 \mathrm{~m}$ high. Simplified Tacha as a combination of cone and cylinder for calculation without changing its overall weight. Finally, the pagoda is regarded as a cantilever structure with fixed at bottom, and the other geometric parameters are modeled according to the measured values. The linear elastic analysis is carried out.

Solid185 unit is selected to build the model. The material parameters are selected from the realistic measured values. Modulus of elasticity is $1078 \mathrm{MPa}$, the density is $1900 \mathrm{~kg} / \mathrm{m}^{3}$, and the Poisson's ratio is 0.15 . The grid is divided reasonably to ensure the element quality. The whole system is modeled from bottom to top, as shown in Figure 1(d).

\section{Modal Analysis}

Through modal analysis of Pagoda in Songyue Temple, the first nine vibration modes 、 natural vibration frequency and natural period of vibration are measured. As shown in Table 1 and Figure 2. The vibration modes show that the first two steps are transverse bending. Others rotate about the longitudinal axis is more remarkable. Vibration modes stabilize after the ninth. There is no abrupt change of stiffness, but the whiplash effect is obvious.
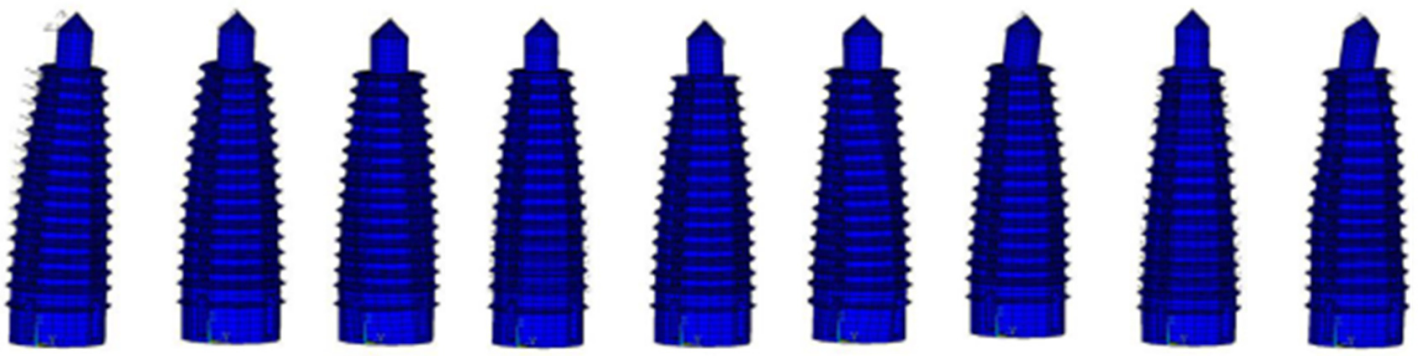

Figure 2. The first nine vibration modes

Table 1. The first nine natural vibration frequency and natural period of vibration

\begin{tabular}{ccc}
\hline No & Natural vibration frequency $(\mathbf{H z})$ & Natural period of vibration $(\mathbf{s})$ \\
\hline 1 & 0.70853 & 1.4114 \\
2 & 2.7457 & 0.3642 \\
3 & 2.8097 & 0.3559 \\
4 & 3.4033 & 0.2938 \\
5 & 5.3519 & 0.1868 \\
6 & 7.0569 & 0.1417 \\
7 & 7.8619 & 0.1272
\end{tabular}




$\begin{array}{lll}8 & 9.8864 & 0.1011 \\ 9 & 10.723 & 0.0933\end{array}$

The modal analysis shows that the first natural period of vibration is $1.4114 \mathrm{~s}$, which is about $10 \%$ different from the result of empirical formula (1) of $1.277 \mathrm{~s}$. Error analysis: due to the simplification of the Tasha and decoration of waist eaves, according to the research experience, the error is within the reasonable range [6 10], which shows that the finite element calculation model is feasible.

$T_{1}=0.0065 \eta_{1} n_{2} n_{3} n_{4} H^{2} / D$ n) _ The influence coefficient of wall thickness, $\eta_{1}=\sqrt{\frac{\left(1-\alpha^{2}\right)}{\left(1-\alpha^{4}\right)}}$ $\alpha=\mathrm{d} / D$

$\eta_{1} \ldots$ _ The influence coefficient ofsection shape, 1.0 ;

$\eta_{1}$ ___ The influence coefficient ofmasonry material,1.0;

$\eta$. The influence coefficient of wall with opening, 1.1 .

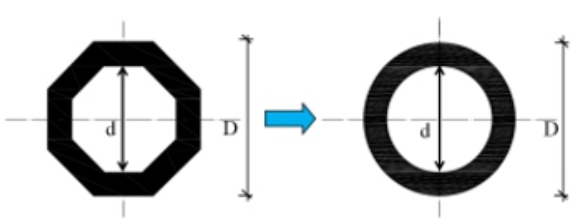

Figure 3. Calculation diagram

\section{Seismic Response}

Combined with response spectrum method and time history analysis method, the seismic response of Pagoda is analyzed with states of stress and deformation. According to the code [11 12], the design value of compressive strength $f_{\mathrm{c}}=2.862 \mathrm{Mpa}$, and the design value of tensile strength $f_{\mathrm{t}}=0.138 \mathrm{Mpa}$, which are taken as the index to judge the damage.

\section{1 response Spectrum Analysis}

The seismic precautionary intensity of pagoda in Songyue temple is VII degree. The design basic acceleration of ground motion is $0.1 \mathrm{~g}$. The design earthquake group is the second group. The site soil type is silt, the construction site is class III and the site design characteristic period is $0.55 \mathrm{~s}$. By inputting horizontal earthquake action, including small, medium and large earthquakes with 7 degrees, find that the dynamic response law is basically consistent. The calculation results are shown in Table 2. The displacement of each floor directly proportional to height. The maximum displacement appears at the top of the pagoda. The maximum stress appears in the upper boundary of the first eaves near the door.

The maximum values of the first principal stress are $0.85 \mathrm{MPa}, 2.46 \mathrm{MPa}$ and $5.34 \mathrm{MPa}$, all exceed 0.138 $\mathrm{MPa}$, indicating that the structure is prone to tensile damage. Expect large earthquake action, the mises stress is $0.76 \mathrm{MPa}$ and $2.19 \mathrm{MPa}$, both less than $2.862 \mathrm{MPa}$, indicating that there will difficultly appear compression damage; Under large earthquake action, the mises stress is $4.76 \mathrm{MPa}$, greater than $2.862 \mathrm{MPa}$, which will cause compression damage according to the specified value (relatively conservative). According to the experimental report, the actual value of brick is $16.3 \mathrm{Mpa}$ so that it will not be damaged actualy.

Table 2. Response spectrum analysis results

\begin{tabular}{cccc}
\hline $\begin{array}{c}\text { Earthquake } \\
\text { action }\end{array}$ & $\begin{array}{c}\text { Maximum displacement } \\
(\mathbf{c m})\end{array}$ & $\begin{array}{c}\text { Maximum of first principal } \\
\text { stress (MPa) }\end{array}$ & Mises stress (MPa) \\
\hline Small & 3.39 & 0.85 & $0.55 \mathrm{E}-3 \sim 0.76$ \\
Medium & 9.74 & 2.46 & $016 \mathrm{E}-2 \sim 2.19$ \\
Large & 21.18 & 5.34 & $0.35 \mathrm{E}-2 \sim 4.76$ \\
\hline
\end{tabular}

Figure 4 indicate that weak link will appear in the local part from the first floor to the fifth floor during small earthquake action. With the increase of earthquake degree, the damaged area spread upward. In the case of small and medium earthquakes, there has different between the two sides along the input direction---the damage area is larger in obverse, the large has not. 


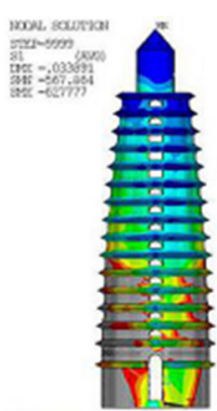

(a) Small earthquake action

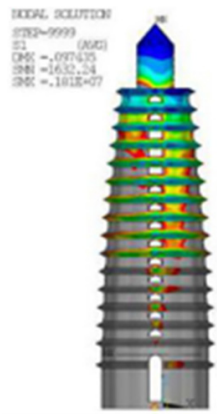

(b) Medium earthquake action

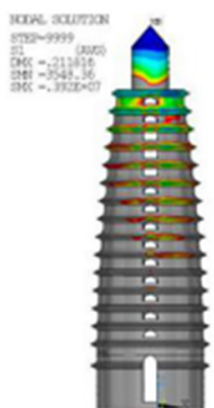

(c) Large earthquake action

Figure 4. Damage characteristic

\subsection{Time history analysis}

The According to the code for seismic design of buildings (GB 50011-2001) [11 12], three seismic waves are selected: El-Centro wave, Taft wave and Tianjin artificial wave. According to 7 degree seismic fortification area, the acceleration peaks of three seismic waves are adjusted to $35 \mathrm{~cm} / \mathrm{s}^{2}, 107 \mathrm{~cm} / \mathrm{s}^{2}$ and $220 \mathrm{~cm} / \mathrm{s}^{2}$. The time interval is $0.02 s$ and the total excitation time is $15 s$. Pagoda in Songyue Temple is a multi-directional symmetrical structure. So only input the north-south axis direction.

\subsubsection{Displacement}

Under the earthquake action of three degree, the displacement response have same law. So there just selacted the displacement nephogram of small to show. The displacement nephogram of three seismic waves show that the displacement response in same height didn't has obviously different, that indicate the mass and stiffness of pagoda in Songyue temple distribute evenly; The top of pagoda's displacement response is the lagest as inputing El-Centro wave and Tianjin artificial wave, but appear at the middle of pagoda as inputing taft wave. As shown in Figure 5. Figure 6 shows that the seimic response of Taft wave is the lagest, Elcentro is the minist. Because the site period of Taft close to the natural period of pagoda easy to introduce remarkable response.

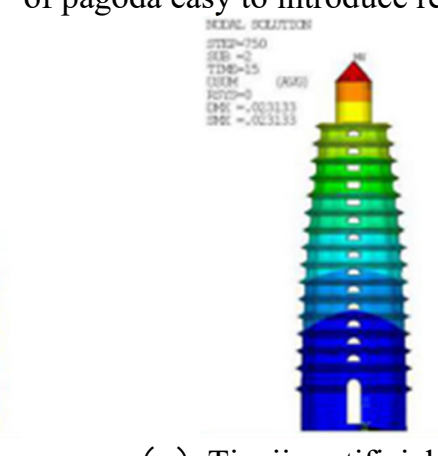

(c) Tianjin artificial

(a) Elcentro

(b) Taft

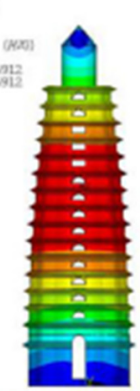

Figure 5. The displacement response of small earthquake action

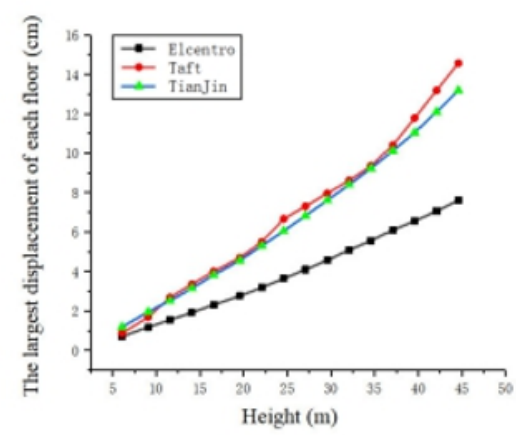

Figure 6. The displacement of each floor 


\subsubsection{Stress results}

As shown in Table 3, the stress response of Tianjin artificial wave is the largest and the Elcentro wave is the smallest. Under the action of three kinds of waves, the maximum value of the first principal stress exceed the material strength $0.138 \mathrm{MPa}$, indicating the tensile strength insufficient. Under small and medium earthquakes action, the mises stress is less than the compressive strength. though it exceeds $2.862 \mathrm{MPa}$ under strong earthquake. According to the experimental report, the actual measured value is $16.3 \mathrm{Mpa}$. So damage will be not caused by compression.

Table 3. The maximum value of the first principal stress ( $\mathrm{MPa})$

\begin{tabular}{cccc}
\hline $\begin{array}{c}\text { Earthquake } \\
\text { action }\end{array}$ & Elcentro wave & Taft wave & Tianjin artificial wave \\
\hline Small & 0.18 & 0.49 & 0.71 \\
Medium & 0.55 & 1.52 & 2.17 \\
Large & 1.13 & 3.14 & 4.46 \\
\hline
\end{tabular}

Taking Tianjin artificial wave as an example. Through comparing the state in Figure 7, it can be seen that under the action of Tianjin artificial wave, the damage degree of head-on and back surface is obviously different along the excitation direction. The damage area is relatively larger than the back. The damage area starts

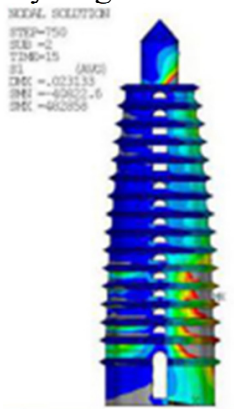

(a) Small earthquake action

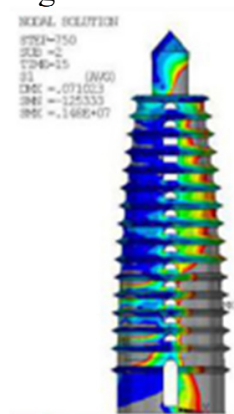

(b) Medium earthquake action at the wall opening and extends to all sides; in the moderate earthquake, the damage has extended to the top; in the large earthquake, the damage is serious. The back side of input direction also has a certain degree of damage.

Figure 7. Damage characteristic

\section{Conclusion}

This paper analyzes the seismic performance of pagoda in Songyue Temple, the dynamic response and damage situation are understood. The conclusions are as follows:

(1) Both time history analysis and spectrum analysis show that the horizontal displacement response increases with height. So does the increment, which accord with the "whiplash effect". In the time history analysis, the stress response under Tianjin artificial wave is the largest; the largest displacement response as input Taft wave and the seismic response under Elcentro wave is the smallest.

(2) The stress results of response spectrum analysis close to it under Tianjin artificial wave. But the damage characteristic has obviously different. By contrast, under the action of Taft wave, the results of seismic response and response spectrum analysis are consistent. The site period similar with Taft wave.

\section{Acknowledgments}

The authors would like to express heartfelt gratitude to the financial supported by the Science Technology of the Ministry of Housing and Urban-Rural Development of the People's Republic of China (2018-K9-065), China Postdoctoral Science Foundation funded project (2018M632805), Kaifeng city key scientific and technological project (2001010), and supported by Foundation of Henan Educational Committee (18A560007).

\section{References}

1. Tong L.p., Tang L., Wang W.H. (2019) Seismic response and the most unfavorable stress state analysis of the pagoda in Songyue Temple[J].World Earth. Eng., 35(02):147-156.

2. Soti R, Abdulrahman L, Barbosa A R, et al. (2020) Case study: Post-earthquake model updating of a heritage pagoda masonry temple 
using AEM and FEM [J]. Eng. Struct., 206:109950.

3. Endo Y , Hanazato T. (2018) Seismic Analysis of a Three-Tiered Pagoda Temple Affected by the 2015 Gorkha Earthquake[J]. Int. J. Architectural Heritage, 1-14.

4. Yuan J. (2013) Study on corresponding relation between seismic intensity and damage degree of ancient masonry pagodas[J]. J. Earth. Eng. and Eng. Vib., 33(2):163-167.

5. WANG J.X., DU Z.F., YAN X.F.,et al. (2016) Evaluation of the seismic behavior of ancient masonry pagoda structure[J]. China encepaper, 11(13):1470-1473.

6. YUAN J.L. (2015) A simplified method for calculating the fundamental period of ancient masonry pagodas[J].Earth. Eng. and Eng. Dyn., 35(02):151-156

7. Song X.B., Wu Y.J., Li K., et al. (2019) Mechanical behavior of a Chinese traditional timber pagoda during construction. Eng. Struct., 196: 109302

8. Yuan J.1.,Fan H., Chen Hanbin,et al. (2005) Experimental study of dynamic behavior of huqiu pagoda[J].Eng. Mech.,22(5): 158-164

9. HOU J.F., SU S.Q., WANG S.L. (2010) Test study of dynamic behavior of an ancient masonry tower [J].Sichuan Building Sci.,36(1): 141-144

10. Chen P.,Yun Z.Y., Song Z.W., (2015) Earthquake risk assessment of ancient masonry tower [J].Ind. Construction,45(04):98-102.

11. GB50003-2011 Code for Design of Masonry Structures [S].Beijing: China Architecture \& Building Press, 2002

12. GB/T50010-2010 Code for design of concrete structures [S].Beijing: China Architecture \& Building Press,2010 